\title{
REPRODUCCIÓN DE LA TARICAYA Podocnemis unifilis TROSCHEL 1848 (TESTUDINES: PODOCNEMIDIDAE) EN LA VÁRZEA DEL MEDIO SOLIMÕES, AMAZONAS, BRASIL.
} Augusto Fachín Terán ${ }^{1}$ y Eduardo Matheus von Mülhen ${ }^{2}$

\section{Resumen}

Este trabajo fue realizado de julio a noviembre de 1998, en la Reserva de Desarrollo Sustentable Mamirauá. La reserva está localizada en la parte media del río Solimões, cerca de la ciudad de Tefé, Amazonas, Brasil. El período de desove de Podocnemis unifilis ocurrió en los meses de agosto y septiembre, y la eclosión en octubre y noviembre. Los nidos fueron construidos en sustratos de greda (67.5\%), arena (25\%), y hojarasca $(7.5 \%)$. La obtención de crías fue mayor en la playa de arena y menor en los barrancos de tierra gredosa. El hombre y la iguana Tupinambis, son los mayores depredadores de huevos. La protección y manejo de los locales de desove, la educación ambiental, la participación comunitaria y la fiscalización permanente del Instituto Brasilero de los Recursos Naturales y del Medio Ambiente - IBAMA son entre las actividades más importantes para viabilizar la recuperación de la población de $P$. unifilis en esta parte de la Amazonía.

Palabras clave: Amazonía, depredación, Podocnemis unifilis, quelonio, reproducción.

\section{Resumo}

Este trabalho foi realizado de julho a novembro de 1998, na Reserva de Desenvolvimento Sustentável Mamirauá. A reserva está situada no médio Solimões, perto da cidade de Tefé, Amazonas, Brasil. O período de desova de Podocnemis unifilis ocorreu nos meses de agosto e setembro, e a eclosão em outubro e novembro. Os ninhos foram construídos em substratos de argila $(67,5 \%)$, areia $(25 \%)$, e folhiço $(7,5 \%)$. A obtenção de filhotes foi maior na praia de areia e menor nos barrancos de terra argilosa. O homem e o lagarto Tupinambis, foram os maiores predadores de ovos. A proteção e o manejo dos locais de desova, a educação ambiental, a participação comunitária e a fiscalização permanente do Instituto Brasileiro dos Recursos Naturais e do Meio Ambiente-IBAMA estão entre as atividades mais importantes para viabilizar a recuperação da população de $P$. unifilis nesta parte da Amazônia.

Palavras chave: Amazônia, Podocnemis unifilis, predação, quelônio, reprodução.

\begin{abstract}
I studied the nesting biology of Podocnemis unifilis from July to November 1998 in the Mamirauá Sustainable Development Reserve located in the Solimões River near Tefé, mazonas, Brazil. Podocnemis unifilis nested in August and September, with the hatching event occurring in October and November. Nests were excavated in clay soils $(67.5 \%)$, sand $(25 \%)$, and leaf litter $(7.5 \%)$. Hatching success was highest in the sand beach nests and lowest in the clay banks nests. Humans and the tegu lizard (Tupinambis) were the main egg predators. This turtle population can be recuperated only by nesting beaches protection, educational programs for the inhabitants of the Reserve, participation of the community in the conservation and management program, and permanent guarding of the nesting beaches by the Instituto Brasilero de
\end{abstract} los Recursos Naturales y del Medio Ambiente personnel.

Kew words: Amazonía, predation, Podocnemis unifilis, turtle, reproduction

\section{Introducción}

La taricaya, Podocnemis unifilis, es un quelonio de tamaño medio dentro de la familia Pelomedusidae. En función de la continua presión de caza a que está siendo sometida, sus poblaciones han disminuido en las áreas donde está distribuida, y actualmente la especie esta en la lista 1, como especie amenazada (UICN, 1996).

En la Reserva de Desarrollo Sustentable Mamirauá -RDSM, esta especie viene siendo permanentemente

\footnotetext{
${ }^{1}$ Universidade do Estado do Amazonas. Escola Normal Superior. Avenida Darcy Vargas 2490 - Bairro Chapada. CEP: 69050-020, Manaus, Amazonas, Brasil. Correo electrónico: fachinteran@yahoo.com.br Universidad Nacional de la Amazonía Peruana. Departamento Académico de Ecología y Manejo. Facultad de Ciencias Biológicas. Casilla Postal 496. Iquitos, Loreto-Perú.

${ }^{2}$ Museu Paraense Emílio Goeldi -Campus de Pesquisa - Centro de Zoologia - Laboratório de Herpetologia-Av. Perimetral, 1901/1907 - Terra Firme - Cx. Postal 399- CEP 66017-970 - Belém, Pará, Brasil. Correo elctrónico: evmreptile@aol.com
} 
explorada para uso en la alimentación de los comunitarios que viven en la reserva; así como comercializada ilegalmente en las ciudades de Tefé y Alvarães (Fachín-Terán, 1999). En esta reserva, la población de $P$. unifilis está seriamente amenazada por el hombre que usa este recurso de manera no sustentable. Según Vogt et al. (1994), uno de los primeros pasos para el manejo y la conservación de cualquier especie es el entendimiento de su ecología reproductiva.

Podocnemis unifilis tal vez sea la especie más investigada dentro de los Podocnemis en lo que se refiere a su ecología reproductiva. Una serie de trabajos fueron realizados en la Amazonía de Brasil (Vanzolini, 1977; Souza \& Vogt, 1994), de Colombia (Medem, 1964; Foote, 1978; Paéz, 1995; Paéz \& Bock, 1997, 1998), de Venezuela (Thorbjarnarson et al., 1993), de Bolivia (Caballero, 1996) y del Perú (Ponce, 1979; Fachín-Terán, 1992, 1993; FachínTerán et al., 1997; Soini, 1995a, 1995b, 1995c; Soini \& Coppula, 1995; Soini \& Soini, 1995a, 1995b, 1995c; Soini, 1996, 1997). A pesar de todo este esfuerzo, el único registro sobre reproducción de esta especie en la várzea del medio Solimões es una nota científica sobre nidificación (Thorbjarnarson \& Silveira, 1996). Casi todos los aspectos sobre la ecología de esta especie son desconocidos en esta parte de la región amazónica.

El propósito de este estudio fue colectar información básica sobre el periodo de nidificación y eclosión, y cuantificar la pérdida de nidos y la sobrevivencia de embriones de $P$. unifilis en la RDSM. Esto ayudará en el futuro a planificar estudios más detallados sobre la ecología de esta especie.

\section{Materiales y métodos}

Nuestro estudio fue realizado en el Sector Jarauá de la Reserva de Desarrollo Sustentable Mamirauá RDSM, que está localizada en la Amazonía Occidental Brasilera, entre los ríos Japurá, Solimões y Auti-Paraná, cerca de la ciudad de Tefé, en el Estado de Amazonas $\left(03^{\circ} 08^{\prime} \mathrm{S}, 64^{\circ} 45^{\prime} \mathrm{W}\right.$ y $2^{\circ} 36$ 'S, 67 $\left.13^{\circ} \mathrm{W}\right)$. Esta reserva tiene una extensión de 1'124,000 ha y está dividida en dos partes: un Área Focal, con cerca de un sexto del área total $(260,000 \mathrm{ha})$, y un Área Subsidiaria complementaria. El Área Focal esta dividida políticamente en nueve sectores. El sector Jarauá esta situado en el río Japurá, y es el mayor de los sectores, con área de $563 \mathrm{~km}^{2}$, y población estimada en 338 habitantes.

La RDSM alberga individuos dispersos de Podocnemis expansa, una pequeña población de $P$. unifilis y una población relativamente grande de $P$. sextuberculata. Existen cuatro especies de quelonios menos abundantes: Phrynops raniceps, Platemys platycephala, Chelus fimbriatus y Kinosternon scorpioides. También ocurre la especie terrestre
Geochelone denticulata, adaptada a las condiciones de la várzea (Fachín-Terán, 1999).

Los nidos de taricaya fueron estudiados en la playa de

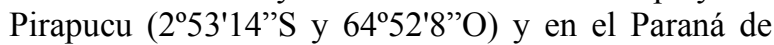
Jarauá (250'52”S y 6458'29”O). Los locales de reproducción fueron visitados periódicamente de julio a noviembre de 1998. Estas áreas fueron identificadas con la ayuda de dos asistentes de campo de la comunidad de San Raimundo de Jarauá. Durante la temporada de desove de 1998, la Playa de Pirapucu fue protegida en común acuerdo entre los comunitarios y el Proyecto Mamirauá, para realizar estudios sobre ecología reproductiva de los quelonios. Algunos nidos encontrados en el Paraná de Jarauá fueron dejados intactos con la finalidad de obtener información in situ sobre el periodo de incubación y cambios en el hábitat en función de la época de vaciante y creciente. Otros fueron transplantados a la Playa de Pirapucu para evitar la depredación por parte del hombre.

Una vez localizado el nido, fueron registradas las siguientes informaciones: fecha de desove, local, distancia del nido a la orilla, tipo de sustrato, diámetro externo del nido, diámetro de la entrada, profundidad y diámetro mayor del nido, número y dimensiones de los huevos. Para determinar el tamaño de los huevos, en nueve nidos se tomó una muestra de 10 unidades, y en dos nidos se tomó la nidada completa. El largo y el ancho de los huevos fue medido con un calibrador vernier cuya precisión era de $1 \mathrm{~mm}$. Dos nidos depredados parcialmente fueron transplantados $\mathrm{y}$ usados en las análisis. En 13 nidos eclosionados, se tomó una muestra de 10 crías, y en dos nidos todas las crías fueron medidas. La biometría de los individuos fue realizada tomándose en cuenta las medidas padrón de largo y ancho del caparazón, y largo del plastrón (Medem, 1976). Todas estas medidas fueron realizadas en línea recta en el punto de mayor amplitud. Los huevos y las crías fueron pesados en balanza Pesola con precisión de $1 \mathrm{~g}$. Después de las mediciones, las crías fueron liberadas próximas de la ribera, en el local donde fue realizado el desove.

Datos del nivel del agua fueron colectados diariamente a las 6:00 am, mediante el uso de escalas móviles, en una base fija situada en la comunidad de San Raimundo de Jarauá.

El ecosistema de várzea alberga una diversidad de tipos de hábitat de los cuales algunos aparecen solo estacionalmente. Algunas denominaciones de estos hábitats solo son usadas localmente y no conseguimos una traducción del portugués al idioma español. A continuación definimos cada uno de estos términos:

Creciente: fase de aumento del nivel de agua en el ciclo hidrológico anual.

Seca: fase mas baja del nivel de agua en el ciclo hidrológico anual. 
Repiquete: aumento temporal del nivel de agua que precede a la creciente principal (oscilaciones del nivel de agua).

Poza: cuerpo de agua formado cuando el nivel del agua disminuye.

Caños: canales que drenan los lagos en la várzea.

Lagos: cochas de la planicie inundada.

Resaca: brazo de cualquier cuerpo de agua (usualmente lago o canal) que termina en la floresta, y que seca casi por completo durante la época de vaciante.

Paraná: ramal del canal principal de un río que forma un brazo que atraviesa la floresta inundada de la várzea, conectándose aguas abajo al mismo cuerpo de agua.

\section{Resultados}

La nidificación de $P$. unifilis en la RDSM, ocurre con la disminución del nivel de agua que expone las playas de arena y barrancos de tierra (Figura 2). Los desoves ocurrieron entre el 21 de agosto y 21 de septiembre. El 72,5\% de posturas ocurrió entre el 21 y 30 de agosto (Figura 1). En ese periodo también se registraron cinco nidos fuera del área de estudio, en la Playa de Santa Luzia de Horizonte en el río Solimões (Fachín-Terán, obs. pers.).

En 23 nidos donde fue registrado el relieve, 16 $(69.6 \%)$ ocurrieron en la parte alta o "meseta" y siete (30.4\%) en terreno inclinado.

La localización de los nidos en sustrato arenoso solo fue posible con el suelo recientemente removido donde el sustrato es más oscuro, cubierto por arena húmeda removida por las hembras. Nidos construidos en sustrato arcillo, externamente tienen diferencia en la coloración con relación al sustrato del alrededor y también presentan rastros de las uñas sobre la cobertura externa del nido. Los nidos de taricaya son excavaciones con una entrada más o menos circular y una galería que se extiende hasta una cámara mayor, donde son depositados los huevos. Las dimensiones de los nidos son presentadas en la tabla 1.

Fueron medidos 207 huevos, siendo el largo medio de $42.4 \pm 0.2 \mathrm{~mm}$, con un rango de 36.2 a $47.8 \mathrm{~mm}$; el ancho medio de $30.7 \pm 0.2 \mathrm{~mm}$, con un rango de 26 a $34 \mathrm{~mm}$; el peso medio de $23.2 \pm 3.5 \mathrm{~g}$, con un rango de 14 a 28 g. Encontramos dos huevos pequeños que midieron 36 × $23.8 \mathrm{~mm}$ y 29.7 x $24.3 \mathrm{~mm}$, y pesaron 10 y 11 gramos respectivamente. Huevos recientes presentaron color rosáceo y forma elíptica, con una secreción pegajosa y transparente.

En 16 nidos incubados en sustrato arenoso, el tiempo medio de incubación de los huevos fue mayor (61.7 \pm 3 días), con respecto a cinco nidos incubados en sustrato arcilloso $(72.8 \pm 0.44$ días).

Fueron medidas 223 crías, siendo el largo medio del caparazón de $43.1 \pm 0.2 \mathrm{~mm}$, con un rango de 37.6 a $48.5 \mathrm{~mm}$; el ancho medio del caparazón fue $38.7 \pm 0.2 \mathrm{~mm}$, con un rango de 33.7 a 43.4; el largo medio del plastrón fue $40.2 \pm 0.2 \mathrm{~mm}$, cun un rango de 32.8 a $44.6 \mathrm{~mm}$; el peso medio fue $16+2.3$ $\mathrm{g}$, rango de 11 a $21 \mathrm{~g}$. Encontramos tres crías pequeñas que midieron entre 32.5 a $33.5 \mathrm{~mm}$, y pesaron de 7 a 8 gramos. En una nidada de 35 huevos que se desarrolló normalmente, todas las crías eran pequeñas.

Figura 1. Período de desove $(\mathrm{N}=40)$ y eclosión $(\mathrm{N}=21)$ en función del nivel del agua, registrados en Podocnemis unifilis, en el sector Jarauá de la RDSM.

Fueron registrados 40 nidos de $P$. unifilis en diferentes locales del sector Jarauá: Resaca de Tucuxi $(\mathrm{N}=11)$, Playa de Pirapucu $(\mathrm{N}=8)$, entrada del Paraná de Jarauá $(\mathrm{N}=8)$, Resaca de Arthur ( $\mathrm{N}=5)$, entrada del caño del Lago Baixo $(\mathrm{N}=4)$, Resaca de Panema $(\mathrm{N}=2)$, Poza de Jaraqui $(\mathrm{N}=1)$, y Playa de Putiri $(\mathrm{N}=1)$.

De los 40 nidos, 27 (67.5\%) fueron depositados en sustrato arcilloso, 10 (25\%) en arena, y tres (7.5\%) en hojarasca. Siete nidos que se encontraban en sustrato arcilloso y que corrían peligro de depredación por parte del hombre fueron transplantados a la playa de Pirapucu en el rió Japura y colocados en sustrato arenoso.
En la muestra de 10 ejemplares medidos, el tamaño medio del largo del caparazón fue de $37.4 \pm 0.08 \mathrm{~mm}$, rango de 35.2 a $38.3 \mathrm{~mm}$; con un peso medio de $7.4 \pm 0.52 \mathrm{~g}, \mathrm{y}$ rango de $7 \mathrm{a} 8 \mathrm{~g}$.

Del total de huevos incubados, $66.8 \%$ eclosionaron. El porcentaje de crías fue mayor en nidos dejados intactos en sustrato arenoso $(92.2 \%)$, que en nidos transplantados $(58.3 \%)$ y nidos dejados intactos en sustrato arcilloso (44.6\%) (Tabla 2). La prueba de Tukey evidencia diferencia estadísticamente significativa con relación a la cantidad de crías obtenidas en función del tipo de sustrato $(\mathrm{p}<0.05)$.

De los 40 nidos, 18 (45\%) permanecieron intactos, $15(37.5 \%)$ fueron depredados por el hombre, cuatro $(10 \%)$ por la iguana Tupinambis y tres $(7.5 \%)$ inundados por un aumento temporal del nivel de las aguas (repiquete). 
Tabla 1. Dimensiones de los nidos, número y tiempo de eclosión de $P$. Unifilis en la RDSM.

\begin{tabular}{lllll}
\hline & $\bar{X}$ & D.E. & RANGO & N \\
\hline Diámetro externo del nido (cm) & 23,4 & 9,6 & $13,5-37,0$ & 10 \\
Diámetro de la entrada del nido (cm) & 11,9 & 2,1 & $9,5-16,0$ & 8 \\
Profundidad hasta el primer huevo (cm) & 8,8 & 1,4 & $6,5-11,0$ & 10 \\
Diámetro mayor de la cámara de huevos (cm) & 16,3 & 2,2 & $13,5-19,0$ & 10 \\
Profundidad total del nido (cm) & 18,2 & 3,1 & $13,5-23,0$ & 10 \\
Distancia del nido a la orilla (m) & 15,9 & 11,5 & $3,0-33,0$ & 22 \\
Número de huevos / nido & 35,1 & 5,9 & $26-49$ & 28 \\
Tiempo de eclosión de los huevos (días) & 64,4 & 5,5 & $56-73$ & 21 \\
\hline
\end{tabular}

$\bar{X}=$ Promedio, D.E. $=$ Desviación estándar, $\mathrm{N}=$ Tamaño de la muestra mes de agosto. Esto ocurre luego que surgen las partes más altas de las playas y de los barrancos en las márgenes de los cuerpos de agua. En esta reserva $P$. sextuberculata concentra sus actividades de nidificación en el inicio del mes de septiembre (Pezzuti, 1998). La especie de mayor tamaño, $P$. expansa desova solamente después que el agua alcanza su nivel más bajo en los meses de septiembre y octubre. Un patrón similar fue observado

Tabla 2. Sobrevivencia y causas de perdida en nidos de P. unifilis en la RDSM.

\begin{tabular}{|c|c|c|c|c|c|c|c|c|}
\hline & \multicolumn{8}{|c|}{ TIPOS DE SUSTRATO } \\
\hline & \multicolumn{2}{|c|}{ ARCILLA } & \multicolumn{2}{|c|}{ ARENA } & \multicolumn{2}{|c|}{ ARCILLA $>$ ARENA } & \multicolumn{2}{|c|}{ TOTAL GENERAL } \\
\hline & $\mathrm{N}=8$ & $\%$ & $\mathrm{~N}=8$ & $\%$ & $\mathrm{~N}=7$ & $\%$ & $\mathrm{~N}=23$ & $\%$ \\
\hline Crías vivas & 117 & 44,6 & 284 & 92,2 & 140 & 58,3 & 541 & 66,8 \\
\hline Huevos sin desarrollo & 32 & 12,2 & 5 & 1,6 & 52 & 21,7 & 89 & 11,0 \\
\hline Huevos comidos por Tupinambis & 10 & 3,8 & 0 & 0,0 & 20 & 8,3 & 30 & 3,7 \\
\hline Embriones atacados por larvas de mosca & 1 & 0,4 & 0 & 0,0 & 19 & 7,9 & 20 & 2,5 \\
\hline Embriones desarrollados - muertos & 8 & 3,1 & 19 & 6,2 & 9 & 3,8 & 36 & 4,4 \\
\hline Embriones muertos por la inundación & 94 & 35,9 & 0 & 0,0 & 0 & 0,0 & 94 & 11,6 \\
\hline TOTAL DE HUEVOS & 262 & 100,0 & 308 & 100,0 & 240 & 100,0 & 810 & 100,0 \\
\hline
\end{tabular}

$\mathrm{N}=$ Número de nidos.

Arcilla Arena $=$ Nidos transplantados de sustrato arcilloso a sustrato arenoso.

\section{Discusión}

Diversos autores observaron que la reproducción de los Pelomedusidae está relacionada al ciclo anual de vaciante y creciente, siendo que el desove y la incubación es realizada en la época de la seca, y el nacimiento de las crías coincide con el inicio de la creciente (Alho \& Pádua, 1982; Fachín-Terán, 1992; Thorbjarnarson et al., 1993; Soini, 1996, 1997). Este mismo patrón fue observado en la RDSM, durante nuestro estudio.

El periodo de desove de $P$. unifilis varía ampliamente a través de la distribución de la especie (Vogt \& Soini, in press). Se puede esperar la existencia de variaciones en los periodos de nidificación en función de las diferencias existentes en las épocas de vaciante y creciente a lo largo de la cuenca Amazónica (Pezzuti, 1998), y la cuenca del Orinoco. Podocnemis unifilis desova en enero y febrero en el rió Capanaparo, Venezuela (Thorbjarnarson et al., 1993); octubre a marzo en el río Caquetá, Colombia (Medem, 1964); julio y agosto en la Reserva Nacional Pacaya-Samiria y en la Reserva de Biosfera del Manú, Perú (Fachín-Terán, 1992; Soini \& Copula, 1995; Mitchell \& Quiñones, 1994); agosto y septiembre en el rio Iténez, Bolivia (Caballero, 1996). En el Brasil el periodo de desove es entre junio y julio en el río Purus, agosto y septiembre en el río negro, río Jaú, río Trombetas, río Tapajós y río de las Muertes (Vogt \& Soini, in press).

Las actividades de nidificación de $P$. unifilis en la RDSM, se concentran durante la última semana del con $P$. expansa en el río Trombetas, Brasil (Alho, 1982) y en el río Pacaya, Perú (Soini, 1997).

Entre los quelonios $P$. unifilis parece ser la especie en que los procesos reproductivos están más adaptados a la diversidad de habitats de la várzea. Esta especie puede desovar en playas de arena, en barro semi-seco y barrancos a las márgenes de los ríos, lagos, paranas y caños (Fachín-Terán, 1992; Souza \& Vogt, 1994; Soini \& Soini, 1995; Caballero, 1996). Los suelos en estés ambientes pueden variar desde arenoso puro, areno-arcilloso, arcilloso amarillo a ceniza, y hojarasca (Fachín-Terán, 1992; Soini \& Soini 1995a). En la RDSM, Thorbjarnarson e Silveira (1996), encontraron en el lago Sanguessuga (= Lago callucallu), un nido construido sobre un colchón de vegetación fluctuante y materia orgánica. El nido fue construido en sustrato compuesto principalmente por hojas en descomposición. Según Vogt \& FloresVillela (1986), especies con amplia distribución geográfica, tienen la plasticidad de comportamiento materno para compensar diferencias climáticas, escogiendo sitios con características distintas o por la desova en época mas caliente o mas fría.

El tamaño medio de la nidada de $P$. unifilis mencionado por diferentes autores varia con la distribución geográfica de la especie. Son 23.3 huevos en Venezuela (Thorbjarnarson et al., 1993); 22.4 (Medem, 1964), 27.3 (Foote, 1978), y 26.8 en Colombia (Páez, 1995); 21 (Ponce, 1979), 23.6 (Landeo, 1997b), 31.3 (Fachín-Terán, 1993) y 34.5 en el Perú (Soini, 1996); 27 en Bolivia (Caballero, 1996); 
$\mathrm{y}$ en Brasil de 20 (Cantarelli \& Herde, 1989), 23 (Valle et al., 1973), 23.7 (Souza \& Vogt, 1994), y 24.4 (Vanzolini, 1977). El tamaño medio de 35.1 huevos encontrado en la RDSM, es el mayor registro para esta especie en el Brasil y solo es semejante al encontrado por Soini (1996) en el río Pacaya, Perú. Los dos locales donde el tamaño medio de la nidada es mayor, son áreas inundables de várzea que renuevan anualmente sus nutrientes a través de los ríos que traen sedimentos de origen andina, siendo muy productivas en términos de nutrientes disponibles.

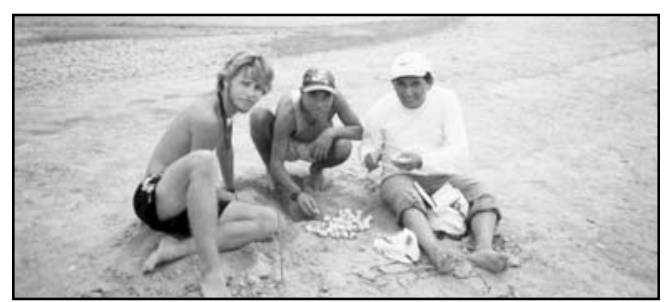

Figura 2. Huevos de Taricaya Podocnemis unifilis en

la Várzea del Medio Solimões, Amazonas, Brasil

La revisión de trabajos que reportan periodo de incubación para $P$. unifilis indican un rango de 55 días (Soini, 1997) a 97 días (Soini \& Coppula, 1995). Generalmente los tiempos más cortos de incubación son obtenidos en nidos construidos en playas de arena con mucha exposición al sol (Thorbjarnarson et al., 1993; Fachín-Terán, 1993; Soini, 1997), y los de mayor periodo de incubación son aquellos construidos en barrancos a las orillas de los ríos, lagos y paranas, donde la vegetación rápidamente cubre el nido y como consecuencia la temperatura de incubación es menor (Fachín-Terán, 1993; Soini \& Coppula, 1995). En el río Caquetá, Colombia, lluvias frecuentes contribuyeron para bajas temperaturas y periodo de incubación mas prolongado en P. unifilis (Paéz \& Bock, 1998). Según Schwarzkopf \& Brooks (1987), y Vogt \& Bull (1982, 1984), las temperaturas de incubación son resultado del clima en determinado año y de las características físicas del nido y del sustrato. En condiciones de cautiverio, playas de arena construidas sobre sustrato húmedo y vegetación arbórea cercana, disminuyen el número de horas de sol en la playa; aumentando el periodo de incubación de los huevos, siendo registrado en $P$. unifilis un tiempo medio de incubación de 112 días con rango de 101 a 136 días (Fachín-Terán et al., 1997).

Altas temperaturas de incubación aceleran el desarrollo embrionario (Bull et al., 1982; Schwarzkopf \& Brooks, 1987; Georges, 1989), por tanto disminuyen el periodo de incubación de los embriones y aumentan la posibilidad de completar su desarrollo (Schwarzkopf \& Brooks, 1987). En este estudio, nidos con menor tiempo de incubación fueron aquellos construidos en sustrato arenoso; en estas condiciones solamente un nido fue cubierto por la vegetación herbácea, lo que aumentó el tiempo de incubación a 69 días. Según Vogt \& Bull (1984) la vegetación puede ser el factor más importante en la determinación de la temperatura del sustrato. Los nidos construidos en sustrato arcilloso tuvieron periodo de incubación mayor, y estaban situados cerca de la vegetación arbórea, siendo cubiertos rápidamente por la vegetación herbácea, lo que influenció en el tiempo de exposición del nido a los rayos solares. Sumado a esto, los lugares de desove en el sustrato arcilloso son más húmedos. Esto puede producir temperaturas más bajas y un mayor tiempo de incubación de las crías, con peligro de que el nido sea alcanzado por la creciente, matando los embriones.

Crecientes repentinas pueden causar grandes pérdidas en los nidos de los quelonios. Soini (1997) cuantifico una pérdida anual de nidos de $P$. unifilis de 10 a $50 \%$ por inundación. La pérdida de nidos de esta especie por inundación en el río Caquetá, Colombia, podría haber sido elevada $(64 \%)$, si los nidos no hubiesen sido transplantados (Paéz \& Bock, 1998). Michell \& Quiñones (1994) señalan que la mayor tasa de perdida natural de nidos de $P$. unifilis, exceptuándose la colecta de los huevos por el hombre, se debe a la inundación adelantada de los sitios de nidificación. Por otro lado, la perdida de nidos puede variar entre años y en función del clima. Landeo (1997b) reportó para el río Manú, la pérdida por inundación de $28.68 \%$ de nidos de $P$. unifilis en 1993 , y de $0.69 \%$ en 1994. Pezzuti (1998) en la RDSM, encontró perdida de $58 \%$ de nidos de $P$. sextuberculata, por el fuerte repiquete durante la seca de 1996. En este estudio, solo tres (7.5\%) nidos fueron inundados por el repiquete. $P$. unifilis desova cuando aparecen las partes superiores de las playas y de los barrancos, lo que puede contribuir para que sus nidos tengan menos posibilidades de ser inundados por la creciente del río.

Soini $(1995,1997)$ reporta que el predador más importante de huevos de $P$. unifilis es el reptil Tupinambis, que posee extrema facilidad para detectar nidos, aunque hayan sido depositados varios días atrás. Fachín-Terán (1994) cita como principal depredador a las hormigas, y Landeo (1997b) cita una especie de grillo. En la RDSM, Tupinambis fue el mayor predador no humano de nidos de $P$. unifilis situados en los barrancos próximos a la floresta (Pezzuti, 1996). Esta observación fue confirmada en este estudio, a través de hallazgos de nidos abiertos y de cáscaras de huevos esparcidos cerca del nido y de la vegetación arbustiva. En las playas de arena no encontramos señales que indicasen la presencia de este animal, ni vestigios de nidos depredados. Esto puede ser debido a que la floresta queda un poco lejos de la playa y a la existencia de una barrera natural constituida por una poza de agua que separa estos hábitats. La presencia constante del personal del proyecto, también puede haber contribuido para que este predador no frecuente la playa. 
En nidos transplantados, $7.9 \%$ de los embriones fueron atacados por larvas de mosca. Vogt et al. (1994) registraron una baja sobrevivencia en crías de Peltocephalus dumerilianus (39.5\%) en función del ataque de larvas de moscas, a las crías que estaban dentro del nido completando su desarrollo. Según Pezzuti (1998) existe la posibilidad de que la manipulación de los huevos pueda facilitar la actividad predatoria de estés dípteros sobre los embriones de quelonios, sugiriendo que seria importante investigar cuando y como la mosca introduce sus huevos en el nido y como ocurre el desarrollo de sus larvas.

La colecta de huevos por las poblaciones indígenas y ribereñas de la Amazonía es una actividad tradicional periódica importante y está relacionada al ciclo reproductivo de los quelonios (Bates, 1863; Soini, 1997; Landeo, 1997a, 1997b). Cuando las playas no son preservadas y existen poblaciones humanas próximas, generalmente la depredación es de $100 \%$ de los nidos de $P$. unifilis por el hombre, como ocurre en el bajo río Jau (Rebêlo et al., 1996). Donde existen programas de preservación, la depredación por el hombre disminuye, y puede variar de local para local y de año para año. Así, en la Reserva Nacional Pacaya-Samiria, Perú, la depredación fue $30.7 \%$ en el río Samiria (Fachín-Terán, 1994) y $37 \%$ en el río Pacaya (Soini \& Coppula, 1995). En el río Manú, Perú, en años diferentes fue registrada una depredación de nidos por el hombre de 39.4 y 52.1\% (Quiñones, 1991; Landeo, 1997a).

La captura de $P$. unifilis y la colecta de sus huevos para consumo por los comunitarios son una amenaza permanente para esta especie en la RDSM, ya que existe un gran número de pescadores utilizando el Paraná de Jarauá. Esta situación se tiende a agravar en función a la implementación desde 1998, del Proyecto de comercialización de Pescado-PCP, que explora comercialmente el recurso pesquero en esta parte de la reserva. Como consecuencia, aumenta la posibilidad de que los pescadores capturen los quelonios en sus redes malladeras. En esta misma reserva, los resultados encontrados para $P$. sextuberculata, sugieren que la pesca con redes malladeras para fines comerciales y la captura de hembras adultas en las playas pueden llevar a una drástica reducción de la población de hembras reproductivas (Fachín-Terán et al., 2003).

La transferencia de nidos de $P$. unifilis a locales protegidos en la RDSM podría ser una opción para producir más crías. Entre tanto en especies donde la temperatura de incubación determina el sexo (e.g. $P$. expansa, Alho et al., 1984; P. unifilis, Souza \& Vogt, 1994; Paéz, 1995; P. sextuberculata, Pezzuti, 1998) esta manipulación debe ser realizada con mucha cautela. Experimentos de manipulación de nidos de tortugas marinas mostraron el peligro de masculinizar la población (Mrosovsky, 1982). Paéz \& Bock (1998) recomendaron que en proyectos de manejo de $P$. unifilis, transferencias de nidos solo deben ser realizados después de pasado el periodo critico de determinación del sexo o transplantados a locales donde la temperatura sea semejante al local inicial. Esto con la finalidad de evitar desvíos para un sexo.

La eclosión de $P$. unifilis en nidos transplantados puede ser alta, pudiendo variar entre 70 a $80 \%$ (Soini, 1995). En este estudio a pesar del cuidado en el traslado de los huevos, el porcentaje de eclosión fue menor $(58.3 \%)$. El relativamente alto número de huevos sin desarrollo (21.7\%) puede ser debido a daños ocurridos en el embrión producidos por la vibración durante el transporte en deslizador. Entre tanto no descartamos la posibilidad de elevada ocurrencia de huevos infértiles.

A pesar del peligro de desvío para un determinado sexo, la transferencia de nidos para locales seguros, parece ser por el momento la única manera de incrementar la población de esta especie; ya que la nidificación ocurre en áreas de exposición permanente para la depredación por el hombre. Esta estrategia es necesaria a pesar de la posibilidad de modificar la proporción de sexos; siendo la única alternativa hasta la realización de acciones de preservación y de estudios mas completos sobre determinación del sexo y dinámica poblacional de esta especie en la RDSM.

La protección y manejo de los locales de desove, la participación comunitaria en la preservación de playas y lagos, la educación ambiental continuada en todos los niveles, la liberación de los ejemplares capturados por los pescadores, y la fiscalización permanente por parte del Instituto Brasilero del Medio Ambiente y de los Recursos Naturales Renovables-IBAMA, en la reserva y en los mercados de las ciudades de Tefé y Alvarães, son acciones importantes para la recuperación de la población de $P$. unifilis en la Reserva Mamirauá.

\section{Agradecimientos}

A la Sociedad Civil Mamirauá-SCM, por el apoyo logístico y facilidades concedidas para la realización de este estudio. Al Dr. José Márcio Ayres, defensor incansable de la várzea, fallecido en marzo de 2003, por darnos la oportunidad de trabajar en la Reserva de Desarrollo Sustentable Mamirauá. A los Drs. John Thorbjarnarson y Richard C. Vogt por la eficiente gestión y soporte en el campo. Al Dr. Renato Cintra, Maria de Fátima Gomes y Souza Soares y Miriam Elenit Lima de Fachín por sus valiosas sugestiones. A Masidonio Pinho de Carvalho, Mariceudo Pinho de Carvalho, Paulo Roberto Barbosa Pereira y Antônio Martins (Presidente) por su valiosa ayuda durante la colecta de datos. Augusto Fachín Terán, recibió una bolsa de doctorado del CAPES, durante su permanencia en el Brasil en el Departamento de Ecología del INPA. Este estudio fue financiado por el 
CNPq/MTC y DFID. Dos revisores dieron valiosas contribuciones al texto final.

\section{Literatura citada}

Alho C.J.R. \& Pádua L.F.M. 1982. Sincronia entre regime de vazante do rio e o comportamento de nidificação da tartaruga da Amazônia Podocnemis expansa (Testudinata, Pelomedusidae). Acta Amazônica. 12: 323-326.

Alho C.J.R., Danni T.M.S. \& Padua L.F.M. 1984. Influência da temperatura de incubação na determinação do sexo da tartaruga da Amazônia Podocnemis expansa (Testudinata, Pelomedusidae). Revista Brasileira de Biologia. 44: 305-311.

Bates H.W. 1863. The naturalist on the river Amazon. John Murray, London.

Bull J.J., Vogt R.C. \& McCoy J. 1982. Sex determining temperatures in turtles: a geographic comparasion. Evolution. 36(2): 326-332.

Caballero J. 1996. Comparación de cuatro tratamientos de manejo para la protección de la peta de agua (Podocnemis unifilis), Troschel 1840, y estimación de la supervivencia de las crías en las playas del río Itenez. Tesis de Licenciado en Ciencias Biológicas. Universidad Autónoma "Gabriel Rene Moreno", Facultad de Ciencias Agrícolas. Santa Cruz de la Sierra-Bolivia.

Cantarelli V.H. \& Herde L.C. 1989. Projeto quelônios da Amazônia 10 anos. Cantarelli, V. H. e L. C. Herde (eds.).Instituto Brasileiro do Meio Ambiente e dos Recursos Naturais Renováveis-IBAMA. Ministério do Interior, Brasília.

Fachín-Terán A. 1992. Desove y uso de playas para nidificación de taricaya (Podocnemis unifilis) en el río Samiria, Loreto-Perú. Boletín de Lima. 79:6575.

Fachín-Terán A. 1993. Características de Podocnemis unifilis (Reptilia, Testudines) en el río Samiria, Loreto. Boletín de Lima. 87: 69-74.

Fachín-Terán A. 1994. Depredación de la taricaya Podocnemis unifilis en la Reserva Nacional Pacaya-Samiria, Loreto. Boletín de Lima. 16(9196): 417-423.

Fachín-Terán A., Acosta A., Vilchez I. \& Taleixo G. 1997. Reproducción de la taricaya Podocnemis unifilis (Reptilia: Testudines) en cautiverio, Iquitos, Perú. En: Tula G. Fang, Richard E. Bodmer, Rolando Aquino y Michael H. Valqui (eds.). Manejo de Fauna Silvestre en la Amazonía, pp.185-189. La Paz, Bolivia.

Fachín-Terán A. 1999. Ecologia de Podocnemis sextuberculata (Testudines, Pelomedusidae), na Reserva de Desenvolvimento Sustentável Mamirauá, Amazonas, Brasil. Tese de Doutorado. Manaus: INPA/UFAM.

Fachín-Terán A., Vogt R.C. \& Thorbjarnarson J.B. 2003. Estrutura populacional, razão sexual e abundância de Podocnemis sextuberculata (Testudines, Podocnemididae) na Reserva de Desenvolvimento Sustentável Mamirauá, Amazonas, Brasil. Phyllomedusa. 2(1): 43-63.

Foote R.W. 1978. Nesting of Podocnemis unifilis (Testudines: Pelomedusidae) in the Colombia Amazon. Herpetologica. 34: 333-339.

Georges A. 1989. Female turtles from hot nets: is the duration of the development of proportion of development that matters?. Oecologia. 81: 323328.

Landeo C. 1997a. Usuarios del recurso taricaya (Podocnemis unifilis) en el río Manú. En: Tula G. Fang, Richard E. Bodmer, Rolando Aquino y Michael H. Valqui (eds.). Manejo de Fauna Silvestre en la Amazonía, pp.181-183. La Paz, Bolivia.

Landeo C. 1997b. Factores limitantes de la población pre-eclosional de la taricaya Podocnemis unifilis en el río Manú. En: Tula G. Fang, Richard E. Bodmer, Rolando Aquino y Michael H. Valqui (eds.). Manejo de Fauna Silvestre en la Amazonía, pp.185-189. La Paz, Bolivia.

Medem F. 1964. Morphologie, Oekologie und Verbreitung der Schildkröte, Podocnemis unifilis in Kolumbiem. Senckenb. Biol. 45: 353-368.

Medem F. 1976. Recomendaciones respecto a contar el escamado y tomar las dimensiones de nidos, huevos y ejemplares de los Crocodylia y Testudines. Lozania. 20: 1-17.

Mitchell C.L. \& Quiñones L. 1994. Manejo y conservación de la taricaya (Podocnemis unifilis) en la Reserva de Biosfera del Manú, Madre de Dios. Boletín de Lima. 16 (91-96): 425-436.

Mrosovsky N. 1982. Sex ratio bias in hatchling sea turtles from artificially incubated eggs. Biol. Conserv. 23: 309-314.

Páez V.P. 1995. The conservation and nesting ecology of the endangered yellow-spotted Amazonian river turtle, Podocnemis unifilis. Ph. D. Thesis, Ohio University, Athens.

Páez V.P. \& Bock B.C. 1997. Nesting ecology of the yellow-spotted river turtle in the Colombian Amazon. In: Van Abemma, J. (Ed.). Proceedings: Conservation, Restoration, and Management of Tortoises and Turtles - An International Conference. New York: New York Turtle and Tortoise Society.: 219-224.

Páez V.P. \& Bock B.C. 1998. Temperature effect on incubation period in the Yellow-Spotted River Turtle Podocnemis unifilis, in the Colombian Amazon. Chelonian Conservation and Biology. 3(1): 31-36

Pezzuti Juarez C.B. 1998. Ecologia reprodutiva da iaçá, Podocnemis sextuberculata (Testudines, Pelomedusidae) na RDSM, Amazonas, Brasil. Dissertação de Mestrado. Manaus, INPA/UFAM. 
Ponce M. 1979. Podocnemis unifilis Troschel 1848 "taricaya" (Chelonia, Pleurodira, Pelomedusidae) en el Bosque Nacional "Alexander von Humboldt”, Loreto-Perú. Tesis de Biólogo. Universidad Nacional Agraria. La Molina, Lima.

Rebêlo G.H., Moreira G., Lugli L., Marajó L., Raposo J.C., Queiroz A.L., Cruz R.F. \& Reimann C. 1996. Os quelônios do Parque Nacional do Jaú (AM). Reporte técnico para a Fundação Vitória Amazônica. (Não publicado).

Schwarzkopf L. \& Brooks J.R. 1987. Nest-site selection and offspring sex ratio in painted turtles, Chrysemys picta. Copeia. 7(1): 55-61.

Soini P. 1995a. Estudio e incubación de los huevos de quelonios acuáticos, 1986. Informe $\mathrm{N}^{\circ}$ 22. En: Soini, P., A. Tovar y U. Valdez (ed.), Reporte Pacaya-Samiria. Investigaciones en Cahuana: 1980-1994, pp. 247-250. CDCUNALM/FPCN/TCN. Lima, Perú.

Soini P. 1995b. Estudio y manejo de quelonios acuáticos, 1987. Informe $\mathrm{N}^{\circ}$ 26. En: Soini, P., A. Tovar y U. Valdez (ed.), Reporte Pacaya-Samiria. Investigaciones en Cahuana: 1980-1994, pp. 279287. CDC-UNALM/FPCN/TCN. Lima, Perú.

Soini P. 1995c. Manejo de quelonios acuáticos, 1992. Informe $\mathrm{N}^{\mathrm{o}}$ 38. In: Soini, P., A. Tovar y U. Valdez (ed.), Reporte Pacaya-Samiria. Investigaciones en Cahuana: 1980-1994, pp. 395-399. CDCUNALM/FPCN/TCN. Lima, Perú.

Soini P. 1996. Reproducción, abundancia y situación de quelonios acuáticos en la Reserva Nacional Pacaya-Samiria, Perú. Folia Amazonica. 8(1): 147164.

Soini P. 1997. Ecología y manejo de quelonios acuáticos en la amazonía peruana. En: Tula G. Fang, Richard E. Bodmer, Rolando Aquino y Michael H. Valqui (eds.). Manejo de Fauna Silvestre en la Amazonía, pp.167-173. La Paz, Bolivia.

Soini P. \& Coppula M. 1995. Estudio, reproducción y manejo de los quelonios del género Podocnemis (charapa, cupiso y taricaya) en la cuenca del Pacaya, río Pacaya, Loreto-Perú. Informe $N^{\circ} 2$. En: Soini, P., A. Tovar y U. Valdez (ed.), Reporte Pacaya-Samiria. Investigaciones en Cahuana: 1980-1994, pp. 3-30. CDC-UNALM/FPCN/TCN. Lima, Perú.

Soini P. \& Soini M. 1995a. Ecología reproductiva de la taricaya (Podocnemis unifilis) y sus implicaciones en el manejo da la especie. Informe No 9. En: Soini, P., A. Tovar y U. Valdez (ed.), Reporte Pacaya-Samiria. Investigaciones en Cahuana: 1980-1994, pp. 99-128. CDCUNALM/FPCN/TCN. Lima, Perú.

Soini P. \& Soini M. 1995b. Ensayos de incubación de huevos de los quelonios del género Podocnemis (charapa, taricaya y cupiso). Informe $\mathrm{N}^{\mathrm{o}} 12$. En: Soini, P., A. Tovar y U. Valdez (ed.), Reporte Pacaya-Samiria. Investigaciones en Cahuana: 1980-1994, pp. 169-176. CDCUNALM/FPCN/TCN. Lima, Perú.

Soini P. \& Soini M. 1995c. Un resumen comparativo de la ecología reproductiva de los quelonios acuáticos. Informe No 19. En: Soini, P., A. Tovar y U. Valdez (ed.), Reporte Pacaya-Samiria. Investigaciones en Cahuana: 1980-1994, pp. 215226. CDC-UNALM/FPCN/TCN. Lima, Perú.

Souza R.R. \& Vogt R.C. 1994. Incubation temperature influences sex and hatchling size in the neotropical turtle Podocnemis unifilis. Journal of Herpetology. 28(4): 453-464.

Thorbjarnarson J.B., Perez N. \& Escalona T. 1993. Nesting of Podocnemis unifilis. Journal of Herpetology. 27 (3): 344-347.

Thorbjarnarson J.B. \& da Silveira R. 1996. Podocnemis unifilis (Yellow-headed Sideneck). Nesting. Herpetological Review. 27(2): 77-78.

IUCN. 1996. Red List of Threatened Animals. Compiled and Edited by Jonathan Baillie and Brian Groombridge. IUCN, Gland, Switzerland.

Valle R.C., Alfinito J. \& da Silva M.M.F. 1973. Contribuição ao estudo da tartaruga Amazônica. Em: Preservação da tartaruga Amazônica. Simpósio Internacional sobre manejo de Fauna Silvestre e Pesca Fluvial e Lacustre Amazónica. IBDF/SUDEPE/IICA, Manaus.

Vanzolini P.E. 1977. A brief biometrical note on the reproductive biology of some South American Podocnemis (Testudines, Pelomedusidae). Papeis Avulsos de Zoologia. 31: 79-102.

Vogt R.C. \& Bull J. 1982. Temperature controlled sex determination in turtles: ecological and behavioral aspects. Herpetologica. 38(1): 156-164.

Vogt R.C. \& Bull J. 1984. Ecology of hatchling sex ratio in map turtles. Ecology. 65(2): 65-74.

Vogt R.C. \& Flores-Villela O. 1986. Determinación del sexo en tortugas por la temperatura de incubación de los huevos. Ciencia. 37: 21-32.

Vogt R.C. 1994. Temperature controlled sex determination as a tool for turtle conservation. Chelonian Conservation and Biology. 1(2): 159162.

Vogt R.C., Cantarelli V.H. \& de Carvalho A.G. 1994. Reproduction of the Cabeçudo, Peltocephalus dumerilianus, in the Biological Reserve of rio Trombetas, Pará, Brazil. Chelonian Conservation and Biology. 1(2): 145-148.

Vogt R.C. \& Soini P. In Press. Podocnemis unifilis Troschel, 1848. Tracajá, Terecay, Yellow-Spotted Amazon Turtle. IUCN/SSC. Conservation Biology of Freshwater Turtles. Vol. II. Eds. A. Rhodin and P. Pritchard. 